\title{
Avaliação da Cavidade Uterina com Hístero-sonografia em Pacientes com Falha de Implantação após Fertilização In Vitro
}

\author{
Evaluation of the Uterine Cavity by Sonohysterography in Patients \\ with Implantation Failure after In Vitro Fertilization
}

\begin{abstract}
Teiichi Ninomiya, Luiz Eduardo Trevisan de Albuquerque, Daniele Mansur Varjão, Karina Bertono de Faria, Rudson Carlos Martins de Oliveira, Luiz Eduardo Vieira Diniz
\end{abstract}

\section{RESUM0}

Objetivo: analisar a acurácia da hístero-sonografia (HSoG) na avaliação da cavidade uterina nas pacientes que tiveram falha de implantação na primeira tentativa de fertilização in vitro. Método: foram analisadas prospectivamente, em estudo duplo-cego, pacientes previamente submetidas à transferência de pelo menos um embrião e que apresentaram falha de implantação. As pacientes foram submetidas a HSoG e em seguida a histeroscopia diagnóstica, realizadas por equipes diferentes, não havendo troca de informações sobre os resultados das mesmas. Os resultados foram agrupados e interpretados somente após o término da pesquisa. A HSoG foi realizada após cateterização do colo uterino por sonda uretral $n^{\circ} 8 e$ injeção de soro fisiológico. A interface anecóica proporcionada pela solução salina permite determinar as anormalidades, tais como pólipos uterinos ou miomas submucosos. A histeroscopia diagnóstica foi realizada com equipamento Karl Storz, óptica rígida de $4 \mathrm{~mm}$ a $30^{\circ}$, e distensão da cavidade uterina com soro fisiológico. Não utilizou-se anestesia local nem houve a necessidade de realizar dilatação cervical.

Resultados: foram estudadas 28 pacientes das 33 inicialmente selecionadas para o presente estudo. A HSoG diagnosticou anormalidades em 8 pacientes, sendo que em cinco pacientes foram encontrados pólipos endometriais (62,5\%), pólipos endocervicais em duas pacientes (25,0\%) e mioma submucoso em uma (12,5\%). A histeroscopia (padrão-ouro) diagnosticou alterações em 7 pacientes, sendo que em duas pacientes (28,6\%) foram encontrados pólipos endometriais, em outras duas pacientes $(28,6 \%)$ pólipos cervicais e em uma paciente, mioma submucoso (14,2\%). A HSoG, quando comparada com a histeroscopia diagnóstica, apresentou sensibilidade de $71,4 \%$, especificidade de $85,7 \%$, valor preditivo positivo de $62,5 \%$ e valor preditivo negativo de $90 \%$.

Conclusões: por apresentar valor preditivo positivo baixo, todas as vezes que a HSoG for anormal, sugerimos confirmar o exame pela histeroscopia diagnóstica. Pelo fato de a HSoG apresentar boa especificidade e bom valor preditivo negativo, poderiamos sugerir que diante de HSoG normal não há necessidade de indicação de se avaliar a cavidade uterina pela histeroscopia diagnóstica antes de realizar a fertilização in vitro. Concluímos que a histerosonografia é bom método para rastreamento de lesões polipóides da cavidade uterina que possam responder pela falha de implantação nas técnicas de fertilização in vitro.

PALAVRAS-CHAVE: Fertilização in vitro. Histero-sonografia. Histeroscopia. Infertilidade.

Correspondência:

Teiichi Ninomiya

Centro de Referência da Saúde da Mulher - Hospital Pérola Byington

Divisão de Reprodução Humana

Av. Brigadeiro Luiz Antônio, 683 - Bela Vista

01317-001 - São Paulo - SP

Fone/Fax: (11) 232-3433

e-mail: teiichi@uol.com.br

\section{Introdução}

Acredita-se que, ao se confirmar a condição de normalidade da cavidade uterina, estamos dando um passo importante para o sucesso pós-transferência de pré-embriões em pacientes submeti- 
das a técnicas de reprodução assistida ${ }^{1}$. Tal fato torna-se evidente, uma vez que alguns autores têm relatado uma incidência de 19 a 50\% de alterações endometriais nas pacientes submetidas à fertilização in vitro $(\mathrm{FIV})^{2,3}$.

O exame mais utilizado para avaliação da cavidade uterina é a histerossalpingografia (HSG), exame que tem como objetivo não só avaliar a cavidade uterina, mas também a permeabilidade e morfologia interna das tubas. A HSG passa a ser um exame desnecessário para as pacientes com indicação de repetição de FIV, quando se deseja observar apenas a cavidade uterina, pois as informações sobre as condições das trompas tornamse desnecessárias, pois a formação dos embriões e sua transferência são feitas em laboratório, mimetizando as tubas uterinas. Por outro lado a histeroscopia diagnóstica, considerada padrão-ouro para a avaliação da cavidade uterina, nem sempre está disponivel para o profissional.

O uso da ultra-sonografia transvaginal para observação da cavidade uterina previamente preenchida com infusão de solução salina, exame também denominado hístero-sonografia (HSoG), passa a ser alternativa interessante, uma vez que tal observação pode ser realizada por qualquer profissional com experiência em exame ultrasonográfico. A HSoG realizada para a detecção de anormalidades da cavidade uterina parece ser exame diagnóstico muito preciso e com excelente sensibilidade e especificidade ${ }^{4,5}$. Ao compararmos a HSG com a HSoG, para o exame da cavidade uterina, devemos considerar que a primeira requer o uso de radiação e não é bem tolerada pela paciente (cólicas), além de apresentar menor sensibilidade diagnóstica. Na comparação com a histeroscopia diagnóstica, devemos considerar que, além de a HSoG ser menos invasiva, é menos onerosa e mais acessivel.

O objetivo do presente estudo é o de observarmos, para os casos nos quais necessitamos verificar as condições apenas da cavidade uterina, como nos casos de insucessos de um ciclo prévio de FIV, se a realização apenas da $\mathrm{HSoG}$ poderia substituir a histeroscopia diagnóstica no rastreamento de lesões endometriais que poderiam comprometer os resultados nas técnicas de reprodução assistida, com vantagens quanto à relação custo-beneficio.

\section{Pacientes e Métodos}

Foram analisadas prospectivamente, em estudo duplo-cego, 33 pacientes regularmente matriculadas no Serviço de Esterilidade Conjugal do Centro de Referência da Saúde da Mulher - Hospital
Pérola Byington, no período de maio a setembro de 2002. As pacientes foram previamente submetidas à transferência de, pelo menos, um embrião classificado como A e/ou $\mathrm{B}^{6}$ e apresentaram falha de implantação. Este estudo foi aprovado pelo Comitê de Ética e Pesquisa do Centro de Referência da Saúde da Mulher - Hospital Pérola Byington.

As pacientes, após assinarem o termo de consentimento informado, foram agendadas para exame de HSoG. No momento da HSoG foram também avaliadas as condições do útero, endométrio e anexos. O aparelho utilizado foi o Aloka $500 \mathrm{com}$ transdutor endovaginal de 7,5 MHz. Após a ultrasonografia a paciente era submetida a exame especular para realizar-se anti-sepsia da cérvix com polivinilpirrolidona-iodo (Povidine) e posteriormente introduzia-se, com ajuda de uma pinça de Cheron, uma sonda uretral $\mathrm{n}^{\circ} 8$ através do orificio externo do canal cervical até a cavidade uterina. A sonda uretral era previamente preenchida com solução salina (soro fisiológico 0,9\%) para minimizar eventuais artefatos de imagem ultrasonográfica (bolhas de ar). Na seqüência, retirava-se cuidadosamente o espéculo vaginal e inseria-se o transdutor transvaginal. Após identificação da cavidade uterina, iniciava-se a instilação do soro fisiológico através da sonda uretral sob visualização ultra-sonográfica contínua.

Após a realização da HSoG as pacientes eram agendadas para avaliação da cavidade por histeroscopia diagnóstica. Neste exame as pacientes foram submetidas, inicialmente, a exame especular para realizar-se anti-sepsia da cérvix com polivinilpirrolidona-iodo (Povidine) e a histeroscopia diagnóstica foi realizada com equipamento Karl Storz com óptica rígida de quatro $\mathrm{mm}$ a $30^{\circ}$, utilizando-se soro fisiológico como meio de distensão da cavidade uterina. Não foi utilizada anestesia local nem a dilatação cervical.

Tanto a HSoG quanto a histeroscopia diagnóstica foram feitas durante a fase folicular do ciclo menstrual. A HSoG e a histeroscopia diagnóstica foram realizadas por equipes diferentes, tomando-se o cuidado de evitar troca de informações sobre os resultados entre as mesmas. Somente após o término da pesquisa é que os resultados foram agrupados e interpretados.

O pólipo endometrial apresenta-se, à ultrasonografia, discretamente mais hiperecogênico em comparação ao endométrio adjacente. Já o mioma submucoso diferencia-se do miométrio por apresentar-se mais hipoecogênico, ou ainda, ecogenicamente heterogêneo, podendo infiltrarse no endométrio, distorcendo a linha da cavidade endometrial.

À visão da histeroscopia diagnóstica, o pólipo endometrial apresenta-se semelhante ao epitélio superficial do endométrio circunvizinho, e de con- 
sistência macia ao contato com a extremidade do histeroscópio. Por outro lado, o mioma submucoso pode apresentar-se com aspecto regular de superfície lisa e recoberto por endométrio homogêneo, semelhante ao resto da cavidade uterina, ou ainda, apresentando superficie branco-perolada, contendo um ou mais vasos sangüineos maiores. Quando na presença de endometrite, forma-se congestão endometrial e os orifícios glandulares tornam-se brancos, dando à superficie endometrial aparência manchada, aspecto idêntico ao observado pela colposcopia na cervicite.

Nenhuma complicação foi relatada durante e após os exames de HSoG e de histeroscopia diagnóstica, exceto cólicas abdominais classificadas como de leve para moderada intensidade, relatadas por algumas pacientes durante e após a realização da histeroscopia diagnóstica.

\section{Resultados}

Das 33 pacientes estudadas, cinco foram excluídas: uma paciente não compareceu ao exame após ter assinado o termo de consentimento informado; em duas pacientes não foi possivel realizar a HSoG por dificuldade em estabelecer e manter uma distensão da cavidade uterina satisfatória, por extravasamento retrógrado em função de alargamento do canal endocervical; em duas pacientes não foi possivel o acesso à cavidade uterina, provavelmente por estenose do orificio interno do canal endocervical.

A HSoG diagnosticou anormalidades em 8 pacientes do total de 28 , e foram encontrados pólipos endometriais em cinco pacientes $(62,5 \%)$, pólipos endocervicais em duas pacientes $(25,0 \%)$ e mioma submucoso em uma paciente $(12,5 \%)$ (Tabela 1).

Tabela 1 - Anormalidades encontradas na hístero-sonografia em 28 pacientes examinadas.

\begin{tabular}{lc}
\hline Diagnóstico & $\mathbf{N}^{\circ}$ de pacientes (\%) \\
\hline Pólipos endometriais & $5(62,5)$ \\
Pólipos endocervicais & $2(25,0)$ \\
Mioma submucoso & $1(12,5)$ \\
\hline
\end{tabular}

A histeroscopia (padrão-ouro) diagnosticou alterações em sete pacientes do total de 28 , e foram encontrados pólipos endometriais em duas pacientes $(28,6 \%)$, pólipos cervicais em duas pacientes $(28,6 \%)$, endometrite em duas pacientes $(28,6 \%)$ e mioma submucoso em uma paciente $(14,2 \%)$ (Tabela 2$)$.
Tabela 2 - Anormalidades encontradas na histeroscopia diagnóstica em 28 pacientes examinadas.

\begin{tabular}{lc}
\hline Diagnóstico & $\mathbf{N}^{0}$ de pacientes $(\%)$ \\
Pólipos endometriais & $2(28,6)$ \\
Pólipos cervicais & $2(28,6)$ \\
Endometrite & $2(28,6)$ \\
Mioma submucoso & $1(14,2)$ \\
\hline
\end{tabular}

Do total de 28 pacientes avaliadas, em 23 $(82,1 \%)$ os exames foram concordantes e em 5 $(17,9 \%)$ discordantes. Nestas cinco, em dois com HSoG normal a histeroscopia diagnóstica revelou presença de endometrite. Nas outras três a HSoG mostrou imagens ultra-sonográficas sugestivas de pólipo endomentrial, sendo uma com imagem corporal alongada de $8 \times 2 \mathrm{~mm}$, a segunda na região cornual de $5 \times 6 \mathrm{~mm}$ e a terceira de $3 \mathrm{~mm}$ em parede posterior não confirmadas pela histeroscopia diagnóstica. Em todos os 20 exames $(71,4 \%)$ em que a HSoG mostrou cavidade endometrial normal, a histeroscopia diagnosticou dois casos de endometrite, confirmando a cavidade endometrial sem lesões polipóides e, portanto, confirmando uma "HSoG normal".

A sensibilidade da HSoG foi de 71,4\% (5/7) e a especificidade foi de $85,7 \%(18 / 21)$. O valor preditivo positivo foi de $62,5 \%(5 / 8)$ e o valor preditivo negativo foi de $90 \%(18 / 20)$ (Tabela 3).

Tabela 3 - Comparação entre os achados da cavidade uterina obtidos pela hísterosonografia HSoG (ShoG) e pela histeroscopia diagnóstica.

\begin{tabular}{lccc}
\hline & Anormal & $\begin{array}{c}\text { Histeroscopia } \\
\text { Normal }\end{array}$ & Total \\
\hline HSoG anormal & 5 & 3 & 8 \\
HSoG normal & 2 & 18 & 20 \\
Total & 7 & 21 & 28 \\
\hline
\end{tabular}

\section{Discussão}

Ainda hoje, a HSG é a técnica preferida e a mais comum para avaliar a cavidade uterina nas pacientes com infertilidade conjugal. Porém, a HSG requer o uso de radiação, além de não ser bem tolerada e apresentar menor sensibilidade diagnóstica. A histeroscopia diagnóstica, considerada padrão-ouro para a avaliação da cavidade uterina, pode não ser bem tolerada pela paciente, tem um custo elevado e nem sempre o aparelho está disponivel para os especialistas.

Este estudo mostrou-se concordante com vários trabalhos que utilizaram a HSoG para a iden- 
tificação de pólipos endometriais. Em 1994, Cicinelli et al. ${ }^{7}$ demonstraram que a HSoG foi tão precisa quanto a histeroscopia diagnóstica para identificar pólipos endometriais.

Não foi o nosso objetivo comparar a ultrasonografia transvaginal com a HSoG, mas foram poucos os exames que apresentaram imagens suspeitas à ultra-sonografia que realizamos previamente à HSoG. Estes dados são comparáveis aos encontrados por Soares et al. ${ }^{8}$, os quais verificaram que a HSoG foi, em geral, o teste mais preciso quando comparado à HSG e à ultra-sonografia transvaginal, considerando-se a histeroscopia diagnóstica como padrão-ouro. Sua precisão diagnóstica foi marcadamente superior nas lesões polipóides (pólipos endometriais e mioma uterino submucoso) e hiperplasias endometriais, com concordância total com o padrão ouro.

Concordamos com Goldberg et al. ${ }^{9}$, que concluíram que a HSoG é método diagnóstico de execução mais fácil, menos dispendioso, mais seguro e melhor tolerado do que a histeroscopia diagnóstica, para as pacientes que apresentam falha de enchimento intra-uterino vista na HSG. Neste trabalho de Goldberg et al. ${ }^{9}$ houve $100 \%$ de correlação entre a HSoG e a histeroscopia. Segundo os autores, a histeroscopia diagnóstica pode ser evitada se a HSoG for normal. A HSoG também pode fornecer informações adicionais sobre a proporção relativa do componente intracavitário (submucoso) e intramiometrial (intramural) dos miomas submucosos, informação que pode ser de grande valia no planejamento cirúrgico para a exérese do tumor.

Kim et al. ${ }^{10}$ constataram que a HSoG oferece vantagem significativa em relação à $\mathrm{HSG}$ e histeroscopia diagnóstica pela sua praticidade, precisão e conforto relativo, ao realizar rastreamento da cavidade uterina prévio à FIV. Apesar de a histeroscopia diagnóstica ser procedimento padrão para rastreamento de anormalidade da cavidade uterina antes da FIV, é invasiva, tem custo elevado e pode causar desconforto, portanto os autores recomendam o uso rotineiro da HSoG.

Pelo fato de a HSoG representar custo menos elevado para o tratamento, ser de fácil realização e pouco invasiva, assim como Alatas et al. ${ }^{11}$, recomendamos o uso da HSoG para o diagnóstico de doenças intra-uterinas em pacientes inférteis. Brown et al. ${ }^{12}$ vão além quando verificaram que a $\mathrm{HSG}$, histeroscopia diagnóstica e a HSoG são estatisticamente equivalentes ao diagnosticar as doenças intra-uterinas em mulheres inférteis. Na avaliação da intensidade de dor durante o procedimento, a HSG e a histeroscopia diagnóstica apresentaram escore médio de dor, o que foi significativamente maior que o escore de dor apresentado na HSoG.
Em nossos resultados, o achado de três exames que mostravam alterações pela HSoG não foi confirmado pela histeroscopia diagnóstica, conseqüentemente foram discordantes. Conforme os dados da literatura ${ }^{13}$, a HSoG realizada na segunda fase do ciclo menstrual pode apresentar alterações sugestivas de pólipo endometrial, imagem que na realidade pode ser o resultado de pequena descamação endometrial. Portanto, os dados apresentados são semelhantes aos encontrados na literatura, pois em nossa casuística as pacientes faziam uso de anticoncepcional hormonal oral contínuo.

Por apresentar valor preditivo positivo baixo $(62,5 \%)$, quando a HSoG for anormal, aconselhamos confirmar o achado pela histeroscopia. Pelo fato de a HSoG apresentar boa especificidade $(85,7 \%)$ e bom valor preditivo negativo $(90,0 \%)$, sugerimos que diante de uma HSoG normal não há necessidade de se realizar a histeroscopia diagnóstica. Apesar da necessidade de novos estudos com maior número de pacientes, concluímos que a HSoG pode ser bom método para rastreamento de lesões polipóides da cavidade uterina que possam responder por falha de implantação nas técnicas de FIV. Em face dos três exames discordantes entre a HSoG e a histeroscopia diagnóstica, sugerimos que a HSoG seja sempre realizada na primeira fase do ciclo (fase proliferativa).

\section{ABSTRACT}

Purpose: to analyze the accuracy of sonohysterography for the evaluation of the uterine cavity in patients with an implantation failure, at the first attempt of an in vitro fertilization cycle.

Methods: in a prospective double blind study, the authors analyzed patients previously submitted to at least one embryo transfer, who presented implantation failures. The patients were submitted to a sonohysterographic examination followed by a diagnostic hysteroscopic examination, carried out by different professionals each of whom was not aware of the results of the other. The results were recorded and only interpreted after the end of the trial. Sonohysterography was performed by the introduction of a urethral catheter 8 into the uterine cervix followed by infusion of physiological saline. The anechoic interface shown by the physiological saline can reveal abnormalities, like uterine polyps or submucosal myomas. Hysteroscopy was performed with a Karl Storz equipment, $4 \mathrm{~mm} 30^{\circ}$ rigid telescope, and infusion of physiological saline for uterine cavity distention.

Results: twenty-eight of the 33 originally selected patients for this study were analyzed. Sonohysterography detected abnormalities in 8 patients, five with endometrial polyps $(62.5 \%)$, two with endocervical polyps $(25.0 \%)$, and one with submucosal myoma (12.5\%). Hysteroscopy (gold 
standard) detected abnormalities in 7 patients, two with endometrial polyps (28.6\%), two with cervical polyps (28.6\%) and one with submucosal myoma (14.2\%). Sonohysterography, when compared with diagnostic hysteroscopy, presented $71.4 \%$ sensibility, $85.7 \%$ specificity, $62.5 \%$ positive predictive value, and $90 \%$ negative predictive value of.

Conclusions: due to its low positive predictive value, the authors suggest confirmation of the sonohysterography result by diagnostic hysteroscopy. Because sonohysterography presents a good level of specificity and a favorable low negative predictive value, the authors suggest that after a normal sonohysterography diagnostic hysteroscopy to evaluate the uterine cavity before in vitro fertlization is not necessary. This study leads to the conclusion that sonohysterography is a good screening method for the detection of polypoid lesions of the uterine cavity, which could be responsible for implantation failures in in vitro fertilization cycles.

KEYWORDS: In vitro fertilization. Sonohysterography. Hysteroscopy. Infertility.

\section{Referências}

1. Ron-El R, Bracha Y, Herman A, et al. Prerequisite work-up of the couple before in-vitro fertilization. Hum Reprod 1992; 7:483-6.

2. Shamma FN, Lee G, Gutmann JN, Lavy G. The role of office hysteroscopy in in vitro fertilization. Fertil Steril 1992; 58:1237-9.

3. Golan A, Ron-El R, Herman A, Soffer Y, Bukovsky I, Caspi E. Diagnostic hysteroscopy: its value in an in-vitro fertilization / embryo transfer unit. Hum Reprod 1992; 7:1433-4.

4. Alatas C, Urman B, Aksoy S, Mercan R, Nuhoglu A. Evaluation of uterine cavity by sonohysterography in women scheduled for intracytoplasmic sperm injection. Hum Reprod 1998; 13:2461-2.

5. Albuquerque LGT. Valor da histero-sonografia na avaliação de cavidade endometrial na mulher com sangramento uterino anormal [dissertação]. Campinas: Univ. Estadual de Campinas; 1997.

6. Veek LL. An atlas of human gametes and conceptuses. $1^{\text {st }}$ ed. New York: Parthenon; 1999. p.46-51.

7. Cicinelli E, Romano F, Anastasio PS, Blasi N, Parisi C. Sonohysterography versus hysteroscopy in the diagnosis of endouterine polyps. Gynecol Obstet Invest 1994; 38:266-71.

8. Soares SR, Barbosa dos Reis MM, Camargos AF. Diagnostic accuracy of sonohysterography, transvaginal sonography, and hysterosalpingography in patients with uterine cavity diseases. Fertil Steril 2000; 73:406-11.

9. Goldberg JM, Falcone T, Attaran M. Sonohysterographic evaluation of uterine abnormalities noted on hysterosalpingography. Hum Reprod 1997; 12: 2151-3.

10.Kim AH, McKay H, Keltz MD, Nelson HP, Adamson GD. Sonohysterographic screening before in vitro fertilization. Fertil Steril 1998; 69:841-4.

11.Alatas C, Aksoy S, Akarsu C, Yakin K, Aksoy S, Hayran M. Evaluation of intrauterine abnormalities in infertile patients by sonohysterography. Hum Reprod 1997; 12:487-90.

12.Brown SE, Coddington CC, Schnorr J, Toner JP, Gibbons W, Oehninger S. Evaluation of outpatient hysteroscopy, saline infusion hysterosonography, and hysterosalpingography in infertile women: a prospective, randomized study. Fertil Steril 2000; 74:1029-34.

13.Cullinan JA, Fleischer AC, Kepple DM, Arnold AL. Sonohysterography: a technique for endometrial evaluation. Radiographics 1995; 15:501-14.

Recebido em: 7/5/2003 Aceito com modificações em: 20/8/2003

\section{ATUALIZE SEUS DADOS PELA HOME PAGE DA FEBRASGO www.febrasg0.org.br no link: mudança de endereço}

